\title{
miRNA expression profiling of 'noninvasive follicular thyroid neoplasms with papillary-like nuclear features' compared with adenomas and infiltrative follicular variants of papillary thyroid carcinomas
}

\author{
Nicla Borrelli ${ }^{1,4}$, Maria Denaro ${ }^{1,4}$, Clara Ugolini ${ }^{2}$, Anello Marcello Poma ${ }^{1}$, Mario Miccoli ${ }^{3}$, \\ Paolo Vitti ${ }^{3}$, Paolo Miccoli ${ }^{1}$ and Fulvio Basolo ${ }^{1}$ \\ ${ }^{1}$ Department of Surgical Pathology, Medical, Molecular and Critical Area, University of Pisa, Pisa, Italy; \\ ${ }^{2}$ Department of Laboratory Medicine, Section of Pathology Azienda Ospedaliero-Universitaria Pisana, \\ Pisa, Italy and ${ }^{3}$ Department of Clinical and Experimental Medicine, University of Pisa, Pisa, Italy
}

\begin{abstract}
Follicular variants of papillary thyroid carcinoma include encapsulated (with or without capsular/vascular invasion) and infiltrative forms, which have different clinical behaviors. The encapsulated forms that lack capsular invasion have an indolent clinical behavior that is similar to benign lesions; therefore, they were recently reclassified as 'noninvasive follicular thyroid neoplasms with papillary-like nuclear features' (NIFTPs). Because NIFTPs have nuclear features of papillary carcinomas, distinguishing between NIFTPs and infiltrative follicular variant of papillary thyroid carcinoma is almost impossible with cytological examination. The aim of this study is to determine whether miRNA expression profiles may help distinguish between NIFTPs versus follicular adenomas and infiltrative follicular variant of papillary thyroid carcinomas. The expression profiling of $\mathbf{7 9 8}$ miRNAs was tested in 54 thyroid tumors, including 18 follicular adenomas, 19 NIFTPs and 17 infiltrative follicular variant of papillary thyroid carcinomas, using nCounter Nanostring. We found that miR-146-5p, miR-221-5p, miR-222-3p, miR-30e-3p, and miR-152-3p could discriminate between benign and malignant lesions with a very high level of significance $(P$-value $<0.001)$. High expression levels of miR-146-5p, miR-199a-5p, miR-199b-5p, miR-1285-5p, miR-1915-3p, and miR-4516, and low miR-148b-3p expression were associated with infiltrative growth of follicular variant of papillary thyroid carcinomas. Interestingly, miR-152-3p, miR-185-5p, and miR-574$3 p$ were significantly downregulated in NIFTPs compared with follicular adenomas, whereas miR-10a-5p and miR-320e can discriminate between NIFTPs and infiltrative forms of follicular variant of papillary thyroid carcinomas. In conclusion, a panel of these markers could have high diagnostic potential as well as could be applied to presurgical fine-needle aspiration, especially for lesions classified as indeterminate thyroid nodules. Modern Pathology (2017) 30, 39-51; doi:10.1038/modpathol.2016.157; published online 2 September 2016
\end{abstract}

Thyroid nodules are very common and, with highresolution ultrasound detection, their incidence has increased to up to $67 \%$ (ranging from 19 to $67 \%$ ) of the population. ${ }^{1}$ The majority of nodules are benign; from 0.5 to 10 cases per 100000 population are

Correspondence: Professor F Basolo, MD, Department of Surgical Pathology, Medical, Molecular and Critical Area, University of Pisa, Via Savi 10, Pisa 56126, Italy.

E-mail: fulvio.basolo@med.unipi.it

${ }^{4}$ These authors contributed equally to this work.

Received 11 May 2016; revised 21 July 2016; accepted 22 July 2016; published online 2 September 2016 diagnosed as differentiated thyroid carcinomas. ${ }^{2}$ Papillary thyroid carcinoma is the most common endocrine malignancy. On the basis of histological features, it can be further subdivided into several variants. Among these the follicular variant represents a heterogeneous group of lesions rather than a single entity. In detail, follicular variant of papillary thyroid carcinomas included different entities: encapsulated forms with or without capsular and/ or vascular invasion and non encapsulated or infiltrative forms with invasion of the surrounding thyroid parenchyma. It is widely accepted that the aggressiveness of these lesions is related to the 
absence of a capsule or to neoplastic capsule invasion and/or the presence of infiltration into surrounding tissue. However, the cytomorphological features of tumors with indolent behavior are not significantly different from those with aggressive phenotypes. $^{3-6}$

The encapsulated follicular variant of papillary thyroid carcinomas have been reported as low-risk tumors with very rare metastasis and recurrence rates, ${ }^{7-9}$ possessing clinical and biological behavior similar to follicular adenomas when noninvasive, and to follicular carcinomas if displaying capsular/ vascular invasion. ${ }^{6,10,11}$

As recently reported by a group of international experts, the noninvasive encapsulated form of follicular variant of papillary thyroid carcinoma has extremely indolent behavior and formally represents a distinct class of thyroid tumors, termed 'noninvasive follicular thyroid neoplasms with papillary-like nuclear features' (NIFTP). ${ }^{12}$

Hence, to reduce the risk of overdiagnosis and, therefore, overtreatment of this subset the distinction between the noninvasive completely encapsulated variant and invasive and/or infiltrative forms was decisive.

Differentiating between follicular adenoma and the noninvasive encapsulated follicular variant of papillary thyroid carcinoma, based on only morphologic, cytological, and clinical features, is sometimes difficult. However, a strong correlation between the cytological and histological nuclear features was recently reported. ${ }^{13}$ Yet, for presurgical fine-needle aspiration, the cytological distinction between noninvasive encapsulated and invasive or infiltrative carcinoma is extremely difficult. Moreover, molecular analysis seems useless because these tumor types harbor mutations that are also present in either follicular adenoma or follicular thyroid carcinoma. ${ }^{12}$

Recent studies have revealed the emerging role of microRNAs (miRNAs) - a class of endogenous noncoding RNA-as negative regulators of proteincoding gene expression. ${ }^{14,15}$ Specific subsets of overexpressed or downregulated miRNAs have been identified in various human cancers, suggesting that aberrations in miRNA expression may be important in tumor development and progression. ${ }^{16-20}$ In addition, a different miRNA expression profile has been shown between tumors and the corresponding unaffected normal tissue as well as between tumors with distinct biological properties. ${ }^{21,22}$

Some studies have reported comparisons of miRNA profiles between papillary thyroid carcinomas and follicular adenomas, multinodular goiter, and normal thyroid tissues. ${ }^{23-25}$ As a result, a number of miRNAs that are significantly upregulated in papillary thyroid carcinomas have been identified; by contrast, several miRNAs have been found to be downregulated in malignant lesions compared with normal thyroid tissue. ${ }^{23,24,26-28}$ Other reports have shown that there is a specific miRNA profile associated with the clinical behavior of papillary thyroid carcinoma. In detail, upregulation of miR-31, miR-146, miR-155, miR-221, and miR-222 and downregulation of miR-1, miR-34b, miR-130b, and miR-138 were observed in the aggressive form of papillary thyroid carcinoma compared with nonaggressive tumors. ${ }^{29}$

On this basis, the present study aimed to delineate the molecular profile of the follicular variant of papillary thyroid carcinomas in noninvasive encapsulated and infiltrative forms to better classify these tumors into biologically and clinically relevant entities.

\section{Materials and methods}

\section{Patients and Study Design}

The study included 18 follicular adenomas and 36 follicular variant of papillary thyroid carcinomas obtained from patients who underwent surgery at the Department of Surgical, Medical, Molecular Pathology and Critical Area of the University of Pisa, Italy, from 2013 to 2015. In the latter group, 19 were diagnosed with NIFTPs and 17 with infiltrative follicular variant of papillary thyroid carcinomas. Tissue samples were fixed in $10 \%$ buffered formalin and embedded in paraffin for routine histopathological examination. Histological sections $(2-4 \mu \mathrm{m}$ thick) were cut and stained with hematoxylin and eosin (Automatic Stainer Varistain Gemini Sheldon). Hematoxylin-eosin-stained sections of patients from the archives of the Section of Pathology of the University of Pisa were independently re-evaluated by two pathologists (CU and FB). A diagnostic concordance rate of $98 \%$ was achieved between the two investigators. Rare, discordant cases were eliminated. Follicular adenomas and infiltrative follicular variant of papillary thyroid carcinomas were classified according to the WHO 2004 histopathological criteria ${ }^{30}$ and noninvasive encapsulated follicular variant of papillary thyroid carcinomas were reconsidered according to Nikiforov et al. ${ }^{12}$ The capsules of all encapsulated cases were re-evaluated (cases without total capsule evaluation were excluded). Noninvasive encapsulated and infiltrative type of follicular variant of papillary thyroid carcinoma are shown in Figure 1.

Institutional approval from the local ethics committee was obtained for this study. Surviving patients gave written informed consent for the use of their tissue specimens in present and future investigations.

\section{miRNA Extraction}

Total RNA, including miRNA, was isolated from formalin-fixed and paraffin-embedded tissue using a miRNeasy Mini Kit (Qiagen, Hilden, Germany) according to the manufacturer's instructions. The RNA concentration was assessed using a NanoDrop 

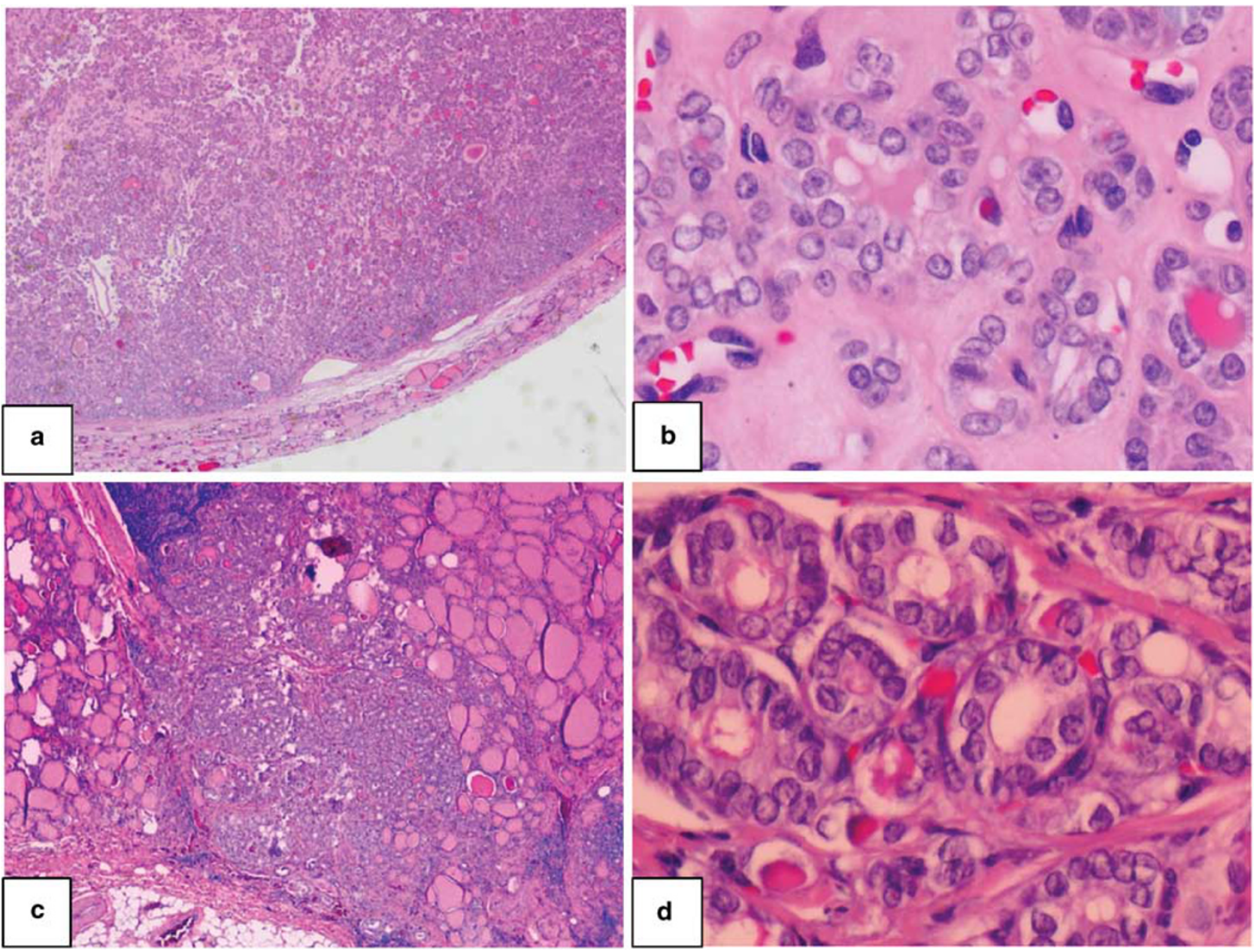

Figure 1 Follicular variant of papillary thyroid carcinoma (a, b) encapsulated type, noninvasive (renamed as noninvasive follicular thyroid tumor with papillary-like nuclear features-NIFTP) ( $\times 2$ and $\times 40$ magnification); (c, d) infiltrative type $(\times 2$ and $\times 40$ magnification),

spectrophotometer (Thermo Scientific, Wilmington, DE, USA).

\section{NanoString nCounter Assay}

The nCounter v3 miRNA Expression Assays used in this study were designed and synthesized by Nanostring Technologies (Nanostring, Seattle, WA, USA).

The miRNA panel consisted of unique oligonucleotide tags onto 798 human miRNAs (from miRBase v21) and five housekeeping mRNAs for reference ( $A C T B, B 2 M, G A P D H, R P L 19$, and RPLPO). Moreover, each assay included 25 control probes that recognize synthetic mRNA or miRNA targets to monitor the efficiency and specificity in each reaction step.

The miRNAs were hybridized using $150 \mathrm{ng}$ of total RNA in addition to the probe pairs consisting of a Reporter Probes, which carry the signal on their $5^{\prime}$ end, and a Capture Probes, which carry biotin on their $3^{\prime}$ end.
Hybridization was performed for $16 \mathrm{~h}$ at $65^{\circ} \mathrm{C}$ in a SensoQuest (SensoQuest, Göttingen, Germany) thermal cycler. Sample cleanup and digital report counts were performed according to the manufacturer's instructions.

Raw data were analyzed using NanoString nSolver version 2.5 software. First, raw data were normalized using internal positive controls to remove variability in the hybridization process and negative controls to subtract the background. In addition, miRNA input levels were normalized using the geometric mean of the top 100 miRNAs with the lower variability coefficients according to the manufacturer's protocol.

\section{DNA Extraction and Genotyping Analysis}

DNA was extracted using the QIAamp DNA Mini Kit (Qiagen, Hilden, Germany) according to the manufacturer's protocol. DNA quantification and quality was tested with NanoDrop Spectrophotometer (Thermo Fisher Scientific, Waltham, MA, USA). 
Mutational status of BRAF (exon 15), NRAS (exons 2 and 3), HRAS (exons 2 and 3), KRAS (exons 2 and 3) was performed using direct DNA sequencing on a 3130 Genetic Analyzer (Applied Biosystems) according to standard procedures. ${ }^{31,32}$

\section{Statistical Analysis}

The Shapiro-Wilk test was performed to verify the normality of the distributions. The Mann-Whitney test, $t$-test, Kruskal-Wallis test and ANOVA test were performed; the Dunn's test and the Bonferroni's test were used for the multiple comparisons. Power analyses were conducted to estimate the sample sizes of the groups. The 1- $\beta$ values of the significant variables were $>0.8$, assuring a low risk of type II error and appropriate sample sizes. The statistical analysis was performed using IBM SPSS software package, version 17.0.1.

\section{Results}

The expression profiling of 798 miRNAs was evaluated using a nCounter Nanostring platform on 54 thyroid tumors, including 18 (33\%) follicular adenomas, 19 (35\%) NIFTPs and 17 (32\%) infiltrative follicular variant of papillary thyroid carcinomas.

Six-hundred-thirty miRNAs with an average count of less than 20 (mean \pm 2 s.d. of negative controls) were excluded to subtract the background noise. The remaining 168 miRNAs were suitable for analyses. Among these, we identified 42 miRNAs with significantly different expression between follicular adenomas and follicular variant of papillary thyroid carcinomas (NIFTPs and infiltrative follicular variant of papillary thyroid carcinomas).

In addition, multiple comparison analysis between histological groups was performed to identify miRNAs that could be associated with a specific subtype or were likely involved in the infiltrative growth of follicular variant of papillary thyroid carcinomas.

\section{miRNAs Differentially Expressed between Follicular Variant of Papillary Thyroid Carcinomas and Follicular Adenomas}

As reported in Table 1, 42 miRNAs had significant deregulation between malignant lesions, follicular variant of papillary thyroid carcinomas (NIFTPs and infiltrative follicular variant of papillary thyroid carcinomas), and benign counterparts (follicular adenomas). Nineteen were upregulated in follicular variant of papillary thyroid carcinomas, whereas 23 were downregulated. In detail, miR-146b-5p, miR-221-5p, and miR-222-3p were highly upregulated $(P$-value $<0.001)$, whereas miR-30e-3p and miR-152$3 p$ were downregulated with the same statistical significance. In addition, we found another 37
Table 1 Deregulated miRNAs in follicular variant of papillary thyroid carcinomas versus follicular adenomas

\begin{tabular}{ll} 
Upregulated & Downregulated \\
\hline $\begin{array}{l}\text { P-value }<0.001 \\
\text { miR-146b-5p } \\
\text { miR-221-5p }\end{array}$ & \\
miR-222-3p & miR-30e-3p \\
& miR-152-3p \\
P-value $<0.01$ & \\
miR-135a-5p & \\
miR-181c-5p & miR-22-3p \\
miR-221-3p & miR-148a-3p \\
miR-551b-3p & miR-185-5p \\
miR-3151-5p & miR-195-5p \\
& miR-204-5p \\
P-value $<0.05$ & miR-574-3p \\
let7c-5p & let-7f-5p \\
miR-31-5p & miR-7-5p \\
miR-100-5p & miR-19b-3p \\
miR-125b-5p & miR-30a-3p \\
miR-130a-3p & miR-30c-5p \\
miR-223-3p & miR-30e-5p \\
miR-302d-3p & miR-148b-3p \\
miR-424-5p & miR-194-5p \\
miR-548ar-5p & miR-200a-3p \\
miR-1915-3p & miR-200b-3p \\
miR-6721-5p & miR-345-5p \\
& miR-362-3p \\
& miR-423-3p \\
& miR-497-5p \\
& miR-518b \\
\hline &
\end{tabular}

miRNAs that were significantly deregulated between these two groups; in 11 , the $P$-value was $<0.01$, whereas in the other 26 , the $P$-value was $<0.05$.

Hierarchical clustering according to differentially expressed miRNAs was performed using nSolver Analysis software with Pearson correlation. This approach separated the groups into two different clusters, 14 out of $18(78 \%)$ follicular adenomas and 26 out of $36(72 \%)$ follicular variant of papillary thyroid carcinomas (Figure 2).

\section{Evaluation of Differential miRNA Expression between NIFTPs, Follicular Adenomas and Infiltrative Follicular Variant of Papillary Thyroid Carcinomas}

NIFTPs versus follicular adenomas. Seven miRNAs exhibited overexpression in NIFTPs compared with follicular adenomas with a very high significant difference in only one miRNA, miR-222-3p. Moreover, 6 miRNAs were upregulated, but they had $P$-values $<0.0167$. By contrast, 13 miRNAs were downregulated, but only miR-152-3p, miR-185-5p, and miR-574-3p had $P$-values $<0.001$ (Table 2).

NIFTPs versus infiltrative follicular variant of papillary thyroid carcinomas. Eight miRNAs showed upregulation and 5 downregulation in infiltrative follicular variant of papillary thyroid carcinomas compared to NIFTPs (Table 3). Among these 


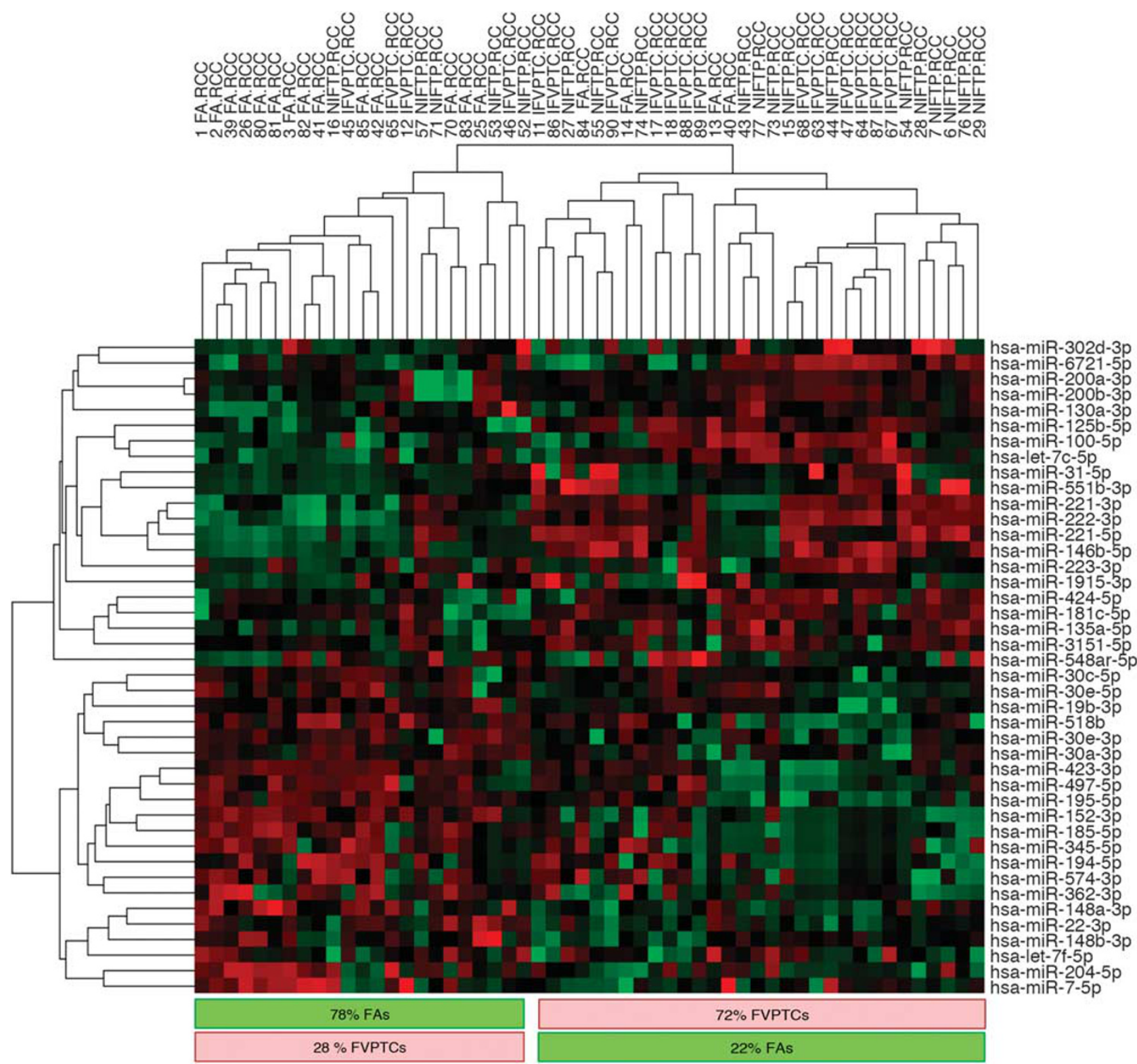

Figure 2 Hierarchical clustering of follicular adenomas and follicular variant of papillary thyroid carcinomas using statistically significant deregulated miRNAs. The columns represent the samples and the rows represent the miRNAs. Only those miRNAs with a different expression statistically significant $(P$-value $<0.05)$ between malignant (noninvasive follicular thyroid tumor with papillary-like nuclear features (NIFTP) and infiltrative follicular variant of papillary thyroid carcinomas) and benign lesions (follicular adenomas) were used for hierarchical clustering. Red and green indicate a high and a low level of expression, respectively. FAs, follicular adenomas; FVPTCs, follicular variant of papillary thyroid carcinomas.

miRNAs, miR-10a-5p, miR-199b-5p, miR-1285-5p and miR-320e were highly significant $(P$-value $<0.001)$.

Infiltrative follicular variant of papillary thyroid carcinomas versus follicular adenomas. In addition to those reported above, we also analyzed and compared miRNA expression in infiltrative follicular variant of papillary thyroid carcinomas and follicular adenomas. Twenty miRNAs had a different pattern of expression; 11 were upregulated and 9 were downregulated in infiltrative follicular variant of papillary thyroid carcinomas. Further details are provided in Table 4.

\section{Differentiating between NIFTPs Versus Follicular Adenomas and Infiltrative Follicular Variant of Papillary Thyroid Carcinomas}

The aim of the following evaluation was to identify miRNAs that are specifically deregulated in different lesions. In particular, we focused on miRNAs that are differentially expressed in NIFTPs versus follicular 
Table 2 Deregulated miRNAs in noninvasive follicular thyroid tumor with papillary-like nuclear features (NIFTP) versus follicular adenomas

\begin{tabular}{ll}
\hline Upregulated & Downregulated \\
\hline $\begin{array}{l}\text { P-value }<0.001 \\
\text { miR-222-3p }\end{array}$ & \\
& miR-152-3p \\
& miR-185-5p \\
miR-574-3p & \\
P-value $<0.0167^{\mathrm{a}}$ & \\
miR-135a-5p & miR-7-5p \\
miR-181c-5p & miR-30e-3p \\
miR-221-5p & miR-194-5p \\
miR-551b-3p & miR-195-5p \\
miR-3151-5p & miR-200b-3p \\
miR-6721-5p & miR-204-5p \\
& miR-296-5p \\
& miR-362-3p \\
& miR-423-3p \\
& miR-497-5p \\
\hline
\end{tabular}

${ }^{\mathrm{a}} \mathrm{P}$-values correct for multiple comparisons (Bonferroni).

Table 3 Deregulated miRNAs in infiltrative follicular variant of papillary thyroid carcinomas versus noninvasive follicular thyroid tumor with papillary-like nuclear features (NIFTP)

\begin{tabular}{ll}
\hline Upregulated & Downregulated \\
\hline P-value $<0.001$ & \\
miR-10a-5p & \\
miR-199b-5p & \\
miR-320e & \\
miR-1285-5p & \\
& \\
P-value $<0.0167^{\mathrm{a}}$ & miR-32-5p \\
miR-132-3p & miR-95-3p \\
miR-199a-5p & miR-148b-3p \\
miR-1915-3p & miR-519d-3p \\
miR-4516 & miR-598-3p \\
& \\
\hline
\end{tabular}

${ }^{\mathrm{a}} \mathrm{P}$-values correct for multiple comparisons (Bonferroni).

Table 4 Deregulated miRNAs in infiltrative follicular variant of papillary thyroid carcinomas versus follicular adenomas

\begin{tabular}{ll}
\hline Upregulated & Downregulated \\
\hline P-value $<0.001$ & \\
miR-146-5p & \\
miR-199b-5p & \\
miR-222-3p & \\
miR-1915-3p & \\
& \\
P-value $<0.0167^{\text {a }}$ & \\
miR-31-5p & let-7f-5p \\
miR-199a-5p & miR-22-3p \\
miR-221-3p & miR-30e-3p \\
miR-221-5p & miR-30e-5p \\
miR-551b-3p & miR-95-3p \\
miR-1285-5p & miR-143-3p \\
miR-4516 & miR-144-3p \\
& miR-148a-3p \\
& miR-148b-3p \\
\hline
\end{tabular}

${ }^{\mathrm{a}} P$-values correct for multiple comparisons (Bonferroni).
Table 5 Specific miRNAs expression in noninvasive follicular thyroid tumor with papillary-like nuclear features (NIFTP) versus follicular adenomas

\begin{tabular}{ll}
\hline Upregulated & Downregulated \\
\hline P-value $<0.001$ & \\
& miR-152-3p \\
& miR-185-5p \\
& miR-574-3p \\
& \\
P-value $<0.0167^{\mathrm{a}}$ & miR-7-5p \\
miR-135a-5p & miR-194-5p \\
miR-181c-5p & miR-195-5p \\
miR-3151-5p & miR-200b-3p \\
miR-6721-5p & miR-204-5p \\
& miR-296-5p \\
& miR-362-3p \\
& miR-423-3p \\
& miR-497-5p \\
\hline
\end{tabular}

${ }^{\mathrm{a}} P$-values correct for multiple comparisons (Bonferroni).

Table 6 Specific miRNAs expression in infiltrative follicular variant of papillary thyroid carcinomas versus noninvasive follicular thyroid tumor with papillary-like nuclear features (NIFTP)

\begin{tabular}{ll}
\hline Upregulated & Downregulated \\
\hline $\begin{array}{l}\text { P-value }<0.001 \\
\text { miR-10a-5p } \\
\text { miR-320e }\end{array}$ & \\
$\begin{array}{ll}\text { P-value }<0.0167^{\mathrm{a}} \\
\text { miR-132-3p }\end{array}$ & \\
& miR-32-5p \\
& miR519d-3p \\
& miR-598-3p \\
\hline
\end{tabular}

${ }^{\mathrm{a}} P$-values correct for multiple comparisons (Bonferroni).

adenomas and in NIFTPs versus infiltrative follicular variant of papillary thyroid carcinomas.

In detail, we found that 16 miRNAs were able to distinguish between NIFTPs and follicular adenomas; we excluded miR-222-3p, miR-221-5p, miR-551b-3p, and miR-30e-3p because their expression levels were also significantly different between follicular adenomas and infiltrative follicular variant of papillary thyroid carcinomas (Table 5).

Moreover, six specific miRNAs (miR-10a-5p, miR-132-3p, miR-320e, miR-32-5p, miR-519d-3p, and miR-598-3p) were capable of dividing infiltrative follicular variant of papillary thyroid carcinomas from NIFTPs, as reported in Table 6; seven miRNAs have been excluded from this analysis because they were deregulated in infiltrative follicular variant of papillary thyroid carcinomas versus follicular adenomas.

The hierarchical clustering analysis showed that 16 out of $18(84 \%)$ and 17 out $18(89 \%)$ of NIFTPs were, respectively, separated from follicular adenomas and infiltrative follicular variant of papillary thyroid carcinomas (Figure 3a and b). 

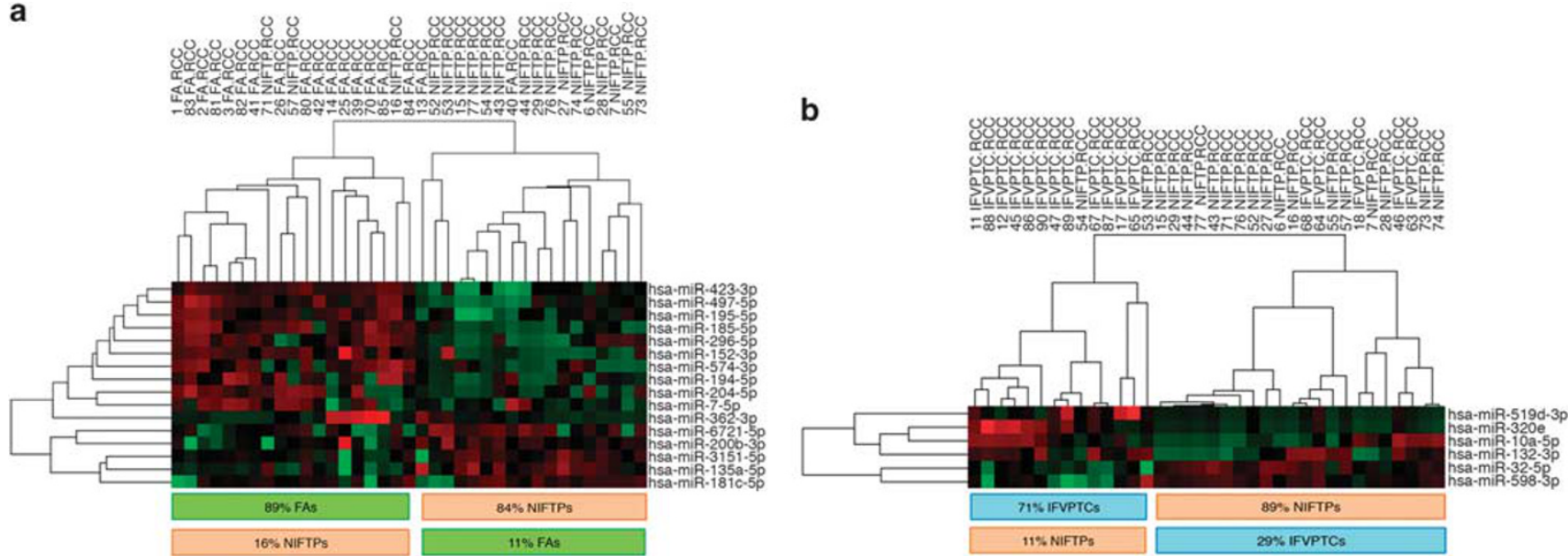

Figure 3 Hierarchical clustering performed using miRNAs expressed with a significant difference between noninvasive follicular thyroid tumor with papillary-like nuclear features (NIFTP) and follicular adenomas (a), noninvasive follicular thyroid tumor with papillary-like nuclear features (NIFTP) and infiltrative follicular variant of papillary thyroid carcinomas (b). FAs, Follicular adenomas; IFVPTCs, infiltrative follicular variant of papillary thyroid carcinomas; miRNA, micro-RNA.

miRNAs Involved in the Infiltrative Growth of Follicular Variant of Papillary Thyroid Carcinomas

In comparing infiltrative follicular variant of papillary thyroid carcinomas with all encapsulated tumors (follicular adenomas and NIFTPs), we observed significant differential expression in 33 miRNAs; 14 were upregulated and 19 downregulated (Table 7).

miR-222-3p and miR-30e-3p were excluded from this list because they were simultaneously significantly deregulated between follicular adenomas and NIFTPs.

As reported in Table 8, considering all results of infiltrative follicular variant of papillary thyroid carcinomas versus encapsulated tumors (infiltrative follicular variant of papillary thyroid carcinomas versus follicular adenomas and infiltrative follicular variant of papillary thyroid carcinomas versus NIFTPs), 33 miRNAs (15 upregulated and 18 downregulated) are likely involved in the infiltrative growth of follicular variant of papillary thyroid carcinomas.

miR-146b-5p, miR-199a-5p, miR-199b-5p, miR-1285$5 p$, miR-1915-3p, and miR-4516 were significantly upregulated $(P$-value $<0.001)$. Among the downregulated miRNAs, only miR-148b-3p was significantly deregulated in infiltrative follicular variant of papillary thyroid carcinomas $(P$-value $<0.001)$.

Moreover, three upregulated (miR-146b-5p, miR-221-3p, and miR-31-5p) and six downregulated miRNAs (miR-22-3p, miR-143-3p, miR-144$3 p$, let-7f-5p, miR-30e-5p, and miR-148a-3p) were differentially expressed when infiltrative follicular variant of papillary thyroid carcinomas were compared with follicular adenomas (Table 8).

When hierarchical clustering analysis was performed using miRNAs involved in infiltrative growth, 36 out of 37 (97\%) encapsulated tumors were grouped in a different cluster with respect to
Table 7 Deregulated miRNAs in infiltrative follicular variant of papillary thyroid carcinomas versus encapsulated tumors

\begin{tabular}{ll} 
Upregulated & Downregulated \\
\hline P-value $<0.001$ & \\
miR-146b-5p & miR148b-3p \\
miR-199a-5p & \\
miR-199b-5p & \\
miR-1285-5p & \\
miR-1915-3p & \\
miR-4516 & \\
& \\
P-value $<0.01$ & \\
miR-10a-5p & \\
miR-222-3p & miR-22-3p \\
miR-320e & miR-95-3p \\
& miR-143-3p \\
P-value $<0.05$ & miR-144-3p \\
miR-10b-5p & \\
miR-21-5p & \\
miR-221-3p & let-7f-5p \\
miR-1290 & miR-25-3p \\
miR-1972 & miR-30e-3p \\
& miR-30e-5p \\
& miR-32-5p \\
& miR-98-5p \\
& miR-126-3p \\
& miR-141-3p \\
& miR-148a-3p \\
& miR-181a-3p \\
& miR-301a-3p \\
& miR-598-3p \\
& miR-1180-3p \\
& \\
&
\end{tabular}

infiltrative follicular variant of papillary thyroid carcinomas (Figure 4).

\section{Genotyping Analysis}

Mutational status of the BRAF and RAS (NRAS, $H R A S$, and KRAS) genes in follicular adenomas, NIFTPs and infiltrative follicular variant of papillary 
Table 8 miRNAs involved in infiltrative growth of follicular variant of papillary thyroid carcinomas

\begin{tabular}{|c|c|c|c|c|c|c|c|}
\hline \multirow{3}{*}{$\begin{array}{l}\text { Upregulated } \\
\text { miRNAs }\end{array}$} & \multicolumn{3}{|c|}{$\begin{array}{c}\text { Infiltrative follicular variant of papillary } \\
\text { thyroid carcinomas versus }\end{array}$} & \multirow{3}{*}{$\begin{array}{l}\text { Downregulated } \\
\text { miRNAs }\end{array}$} & \multicolumn{3}{|c|}{$\begin{array}{c}\text { Infiltrative follicular variant of papillary } \\
\text { thyroid carcinomas versus }\end{array}$} \\
\hline & $\begin{array}{c}\text { Follicular } \\
\text { adenomas } \\
+ \text { noninvasive } \\
\text { follicular thyroid } \\
\text { tumor with } \\
\text { papillary-like } \\
\text { nuclear features }\end{array}$ & $\begin{array}{l}\text { Follicular } \\
\text { adenomas }\end{array}$ & $\begin{array}{l}\text { Noninvasive } \\
\text { follicular } \\
\text { thyroid tumor } \\
\text { with papillary- } \\
\text { like nuclear } \\
\text { features }\end{array}$ & & $\begin{array}{c}\text { Follicular } \\
\text { adenomas } \\
+ \text { noninvasive } \\
\text { follicular thyroid } \\
\text { tumor with } \\
\text { papillary-like } \\
\text { nuclear features }\end{array}$ & $\begin{array}{l}\text { Follicular } \\
\text { adenomas }\end{array}$ & $\begin{array}{l}\text { Noninvasive } \\
\text { follicular } \\
\text { thyroid tumor } \\
\text { with papillary- } \\
\text { like nuclear } \\
\text { features }\end{array}$ \\
\hline & P-value & $\mathrm{P}$-value ${ }^{\mathrm{a}}$ & $\mathrm{P}$-value ${ }^{\mathrm{a}}$ & & P-value & $\mathrm{P}$-value ${ }^{\mathrm{a}}$ & $\mathrm{P}$-value ${ }^{\mathrm{a}}$ \\
\hline $\begin{array}{l}\text { miR-199b-5p } \\
\text { miR-1915-3p } \\
\text { miR-199a-5p } \\
\text { miR-4516 } \\
\text { miR-1285-5p } \\
\text { miR-10b-5p } \\
\text { miR-1290 } \\
\text { miR-21-5p } \\
\text { miR-1972 } \\
\text { miR-146b-5p } \\
\text { miR-221-3p } \\
\text { miR-31-5p } \\
\text { miR-10a-5p } \\
\text { miR-320e } \\
\text { miR-132-3p }\end{array}$ & $\begin{array}{l}<0.001 \\
<0.001 \\
<0.001 \\
<0.001 \\
<0.001 \\
<0.05 \\
<0.05 \\
<0.05 \\
<0.05 \\
<0.001 \\
<0.05 \\
<0.01 \\
<0.01\end{array}$ & $\begin{array}{l}<0.001 \\
<0.001 \\
<0.0167 \\
<0.0167 \\
<0.0167 \\
\\
<0.001 \\
<0.0167 \\
<0.0167\end{array}$ & $\begin{array}{l}<0.001 \\
<0.0167 \\
<0.0167 \\
<0.0167 \\
<0.001\end{array}$ & $\begin{array}{l}\text { miR-148b-3p } \\
\text { miR-95-3p } \\
\text { miR-25-3p } \\
\text { miR-98-5p, } \\
\text { miR-126-3p } \\
\text { miR-141-3p } \\
\text { miR-181a-3p } \\
\text { miR-301a-3p } \\
\text { miR-1180-3p } \\
\text { miR-22-3p } \\
\text { miR-143-3p } \\
\text { miR-144-3p } \\
\text { let-7f-5p } \\
\text { miR-30e-5p } \\
\text { miR-148a-3p } \\
\text { miR-32-5p } \\
\text { miR-519d-3p } \\
\text { miR-598-3p }\end{array}$ & $\begin{array}{l}<0.001 \\
<0.01 \\
<0.05 \\
<0.05 \\
<0.05 \\
<0.05 \\
<0.05 \\
<0.05 \\
<0.05 \\
<0.01 \\
<0.01 \\
<0.01 \\
<0.05 \\
<0.05 \\
<0.05 \\
<0.05 \\
<0.05 \\
<0.05\end{array}$ & $\begin{array}{l}<0.0167 \\
<0.0167 \\
<0.0167 \\
<0.0167 \\
<0.0167 \\
<0.0167\end{array}$ & $\begin{array}{l}<0.0167 \\
<0.0167 \\
<0.0167\end{array}$ \\
\hline
\end{tabular}

${ }^{\mathrm{a}} P$-values correct for multiple comparisons (Bonferroni).

thyroid carcinomas was tested. In detail, 17 out of 18 (94.4\%) follicular adenomas were wild type for $B R A F$ (exon 15) and NRAS, HRAS and KRAS (exons 2 and 3) genes, whereas $1(5.6 \%)$ had BRAF K601E mutation; 9 out of $19(47.4 \%)$ NIFTPs were mutationnegative, 8 out of $19(42.1 \%)$ were mutated for $R A S$ genes (5 NRAS Q61R and 3 HRAS Q61R), and 2 out of $19(10.5 \%)$ had a $B R A F$ K601E mutation; finally, as regards infiltrative follicular variant of papillary thyroid carcinomas, we found that 7 out of 17 $(41.2 \%)$ were wild type for $B R A F$ (exon 15) and NRAS, HRAS, and KRAS (exons 2 and 3) genes, 5 out of $17(29.4 \%)$ were mutated for $R A S$ genes (4 NRAS Q61R and 1 HRAS Q61R), 4 out of 17 (23.5\%) had a $B R A F$ V600E mutation and 1 was not evaluable.

\section{Discussion}

Follicular variant of papillary thyroid carcinomas can have very different clinical behavior and outcomes. Differentiating between follicular variant of papillary thyroid carcinomas (in particular encapsulated forms) and follicular adenoma is sometimes a challenge when considering only the morphological, cytological, or clinical features.
It is extensively reported that completely encapsulated follicular variant of papillary thyroid carcinoma is a very low-risk form (3-5).

One recent noticeable change in thyroid pathology has formally proposed reclassifying noninvasive encapsulated follicular variant of papillary thyroid carcinomas as 'NIFTPs.' 12

For presurgical fine-needle aspiration, the cytological distinction between NIFTP versus invasive and infiltrative follicular variant of papillary thyroid carcinoma has been very difficult, although it was recently observed that there is a good correlation between the cytological and histological nuclear features. ${ }^{13}$ Therefore, to reduce the number of preoperative indeterminate lesions as much as possible, analysis of mutations and rearrangements of specific genes involved in thyroid tumorigenesis have been reported by several studies. ${ }^{33,34}$

Nikiforov's group has extensively reported that improved management for patients who bear thyroid nodules with an indeterminate cytology is now possible using a multi-gene panel of molecular markers with high sensitivity and specificity. ${ }^{30}$ Nevertheless, the molecular test results should be interpreted in the context of cytological, clinical, and ultrasonographic examinations. ${ }^{35}$

In combination, these reports indicate that the addition of extensive molecular characterization to strictly morphological criteria significantly 


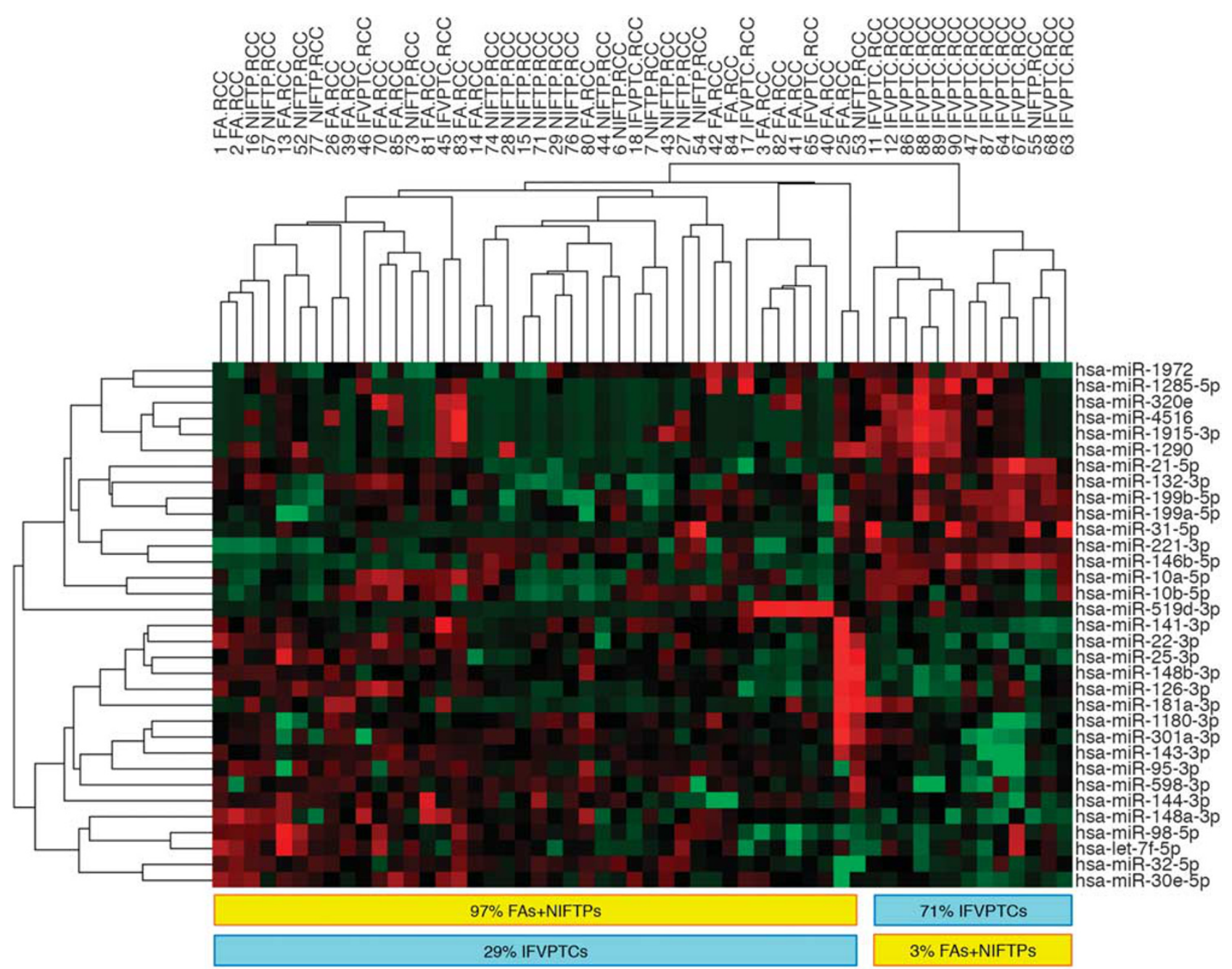

Figure 4 Hierarchical clustering of encapsulated lesions (noninvasive follicular thyroid tumor with papillary-like nuclear features (NIFTP) and follicular adenomas) and infiltrative follicular variant of papillary thyroid carcinomas using significant miRNAs involved in infiltrative growth. FAs, Follicular adenomas; miRNA, micro-RNA; IFVPTCs, infiltrative follicular variant of papillary thyroid carcinomas.

increases the diagnostic accuracy of follicular lesions in $>80 \%$ (CI: $88-97 \%$ ) of the cases. Therefore, $\sim 20 \%$ of patients undergo unnecessary surgery. ${ }^{33}$

Furthermore, mutational analysis is not useful for discriminating between NIFTP versus other follicular-patterned thyroid tumors (follicular carcinomas and follicular adenomas) because of their similar mutational status. ${ }^{12}$

Several studies have evaluated the miRNA profiles of thyroid tumors, highlighting that the miRNA signature could be useful (a) for diagnosis because several miRNAs are deregulated in papillary thyroid carcinoma compare to normal thyroid tissue and (b) for prognosis because miRNA profiles differ in papillary thyroid carcinomas that have different clinical behavior. ${ }^{23,24,26-28}$ Although most prior observations have focused on miRNA expression in papillary thyroid carcinomas, some reports have revealed several miRNA expression differences between follicular carcinoma and follicular adenoma. ${ }^{36,37}$
Interestingly, in situ hybridization analysis has revealed that miR-146b-5p is mainly expressed in papillary thyroid carcinoma, including follicular variant of papillary thyroid carcinoma and is not expressed in most follicular, poorly differentiated, and anaplastic thyroid carcinoma samples. ${ }^{38}$

A study by Dettmer et al. showed that miR-146b is consistently overexpressed in both the classical and follicular variants of papillary thyroid carcinoma; ${ }^{39}$ moreover, others have reported a correlation between miR-146b deregulation and tumor aggressiveness. ${ }^{29,40}$

miR-146b-5p positively regulates the migration and invasion of normal thyroid and tumor follicular cells through a mechanism involving the actin cytoskeleton. $^{41}$ Consequently, miR-146 could be involved in thyroid tumorigenesis considering its important role as a regulator of NF-kb, ${ }^{42,43}$ although this mechanism is not completely understood. In addition, it has recently been found that the miRNA-146 family could interfere with the $R A R b$ gene, which seems to be involved in promoting 
tumor growth and the inefficiency of retinoic acid and radioactive iodine treatment. ${ }^{26}$

In our study, we found that miR-146b-5p was upregulated in malignant lesions compared to benign tumors. Furthermore, miR-146b-5p seems to be specifically useful in differentiating between the infiltrative form of follicular variant of papillary thyroid carcinomas and follicular adenomas and could be a marker of papillary thyroid carcinoma and infiltrative lesions in presurgical fine-needle aspiration.

Other miRNAs, namely miR-221 and miR-222, seem to have important roles. The deregulation of these miRNAs has been reported in several human cancers (colorectal carcinoma, non-small cell lung carcinoma, breast cancer, pancreatic carcinoma, glioma, hematological malignancies, and thyroid cancer, and so on). ${ }^{44}$

Other different studies have consistently reported the upregulation of miR-221 and miR-222 in thyroid cancer, suggesting that this phenomenon is crucial to thyroid carcinogenesis. The finding that these two miRNAs modulate growth receptors, transcription factors and tumor suppressor genes as well as target genes involved in cell growth and apoptosis may explain, at least in part, their role in tumorigenesis. ${ }^{4}$

We confirmed these data, validating that miR-221$5 p$ and miR-222-3p are upregulated in follicular variant of papillary thyroid carcinomas compared with follicular adenomas. Their upregulation has also been observed in NIFTP compared with follicular adenomas as well as in infiltrative follicular variant of papillary thyroid carcinomas compared to follicular adenomas. These data strongly suggest that miR-221-5p and miR-222-3p may be markers of malignancy.

It has been reported that a group of miRNAs is downregulated in malignant lesions compared to normal tissue and benign counterparts, ${ }^{27,28}$ suggesting that both overexpression and downregulation of miRNAs have important roles in tumorigenesis. In human tumors, miR-30e has been reported to be downregulated and associated with advanced carcinoma, such as anaplastic thyroid carcinoma and advanced breast cancer. ${ }^{45,46}$ Many functional mechanisms have been proposed, including miR-30e's role in the mesenchymal-epithelial transition ${ }^{27}$ or, more recently, in suppressing proliferation of hepatoma cells via targeting prolyl-4-hydroxylase subunit alpha-1 (P4HA1) mRNA. ${ }^{47}$ In addition, in our study, miR-30e-3p was significantly downregulated in follicular variant of papillary thyroid carcinomas compared with follicular adenomas, which strongly correlated with the malignancy of the lesion. Another study demonstrated significant downregulation of miR-152, together with other miRNAs (miR-34a, miR-424, and miR-20b) and genes (CCNE2, COL4A1, TRAF6, and HSP9OB1), in papillary thyroid carcinoma and found evidence for its association with aggressive clinical features. ${ }^{48}$ Likewise, we observed significant downregulation of miR-152-3p in follicular variant of papillary thyroid carcinomas compared with follicular adenomas.

The main aim of our study was to identify a group of molecular markers that are capable of discriminating between encapsulated lesions (NIFTP and follicular adenomas) versus infiltrative forms of follicular variant of papillary thyroid carcinoma, and NIFTP versus either follicular adenomas or infiltrative follicular variant of papillary thyroid carcinomas both in histology and cytology. This is a critical point because, according to a new definition of NIFTP, patients carrying this lesion type could be treated in a conservative manner.

For this reason, we analyzed the miRNA expression profile of follicular adenomas together with NIFTP versus the infiltrative form of follicular variant of papillary thyroid carcinomas. We identified several miRNAs, including miR-146-5p, miR-199a, miR199b, miR-1285-5p, miR-1915-3p, miR-4516, and miR-148b$3 p$, that are highly deregulated (some upregulated and others downregulated) and could discriminate between encapsulated lesions (follicular adenomas and NIFTP) and infiltrative follicular variant of papillary thyroid carcinomas.

Recently, Aragon Han et $a l^{49}$ reported that the expression levels of several miRNAs, including miR-199b, were significantly associated with aggressive features of thyroid cancer, such as a large tumor size, extrathyroidal extension, multifocality, lymphovascular invasion, lymph node metastases, and distant metastasis. Conversely, no data have been thus far reported in thyroid tumors for miR-148b, miR-1285-5p and miR-4516, although deregulation of these miRNAs is associated with other cancer types. ${ }^{50-54}$

Taken together, our data suggest that evaluating miR-146-5p, miR-199a, miR199b, miR-1285-5p, miR1915-3p, miR-4516, and miR-148b could be a useful diagnostic tool for differentiating between encapsulated tumors and other more aggressive entities.

In addition to these five miRNAs, we also observed several additional miRNAs (Table 7) that were differentially expressed in the two groups (encapsulated lesions versus infiltrative follicular variant of papillary thyroid carcinomas) albeit with a lower level of significance.

Furthermore, our results suggest that specific miRNA expression patterns could allow us to discriminate between NIFTP and both follicular adenomas and infiltrative follicular variant of papillary thyroid carcinomas. In detail, miR-152-3p, miR-185-5p, and miR-574-3p are strongly downregulated in NIFTP compared to follicular adenomas. The importance of miR-185-5p and miR-574-3p has also been demonstrated in other human tumors, such as non-small cells lung cancer and breast cancer. ${ }^{55-57}$

In the comparison between infiltrative follicular variant of papillary thyroid carcinomas and NIFTP, among six deregulated miRNAs, miRNA-10a-5p and miR-320e were highly upregulated, demonstrating their involvement in a more aggressive form of 
follicular variant of papillary thyroid carcinomas. Nevertheless, high expression of miR-10a has been already associated with lymph node metastasis in non-small cells lung cancer through direct targeting of the PTEN/AKT/ERK signaling pathway, ${ }^{58}$ whereas miR-320e has been reported as a novel prognostic biomarker associated with adverse clinical outcomes in colorectal cancer patients treated with adjuvant chemotherapy. ${ }^{59}$

In conclusion, our data provide evidence for the diagnostic potential of a specific set of miRNAs. In detail, we have shown that there is differential expression of miR-146-5p, miR-221-5p, miR-222-3p, miR-30e-3p, and miR-152-3p in follicular adenomas versus NIFTP and infiltrative follicular variant of papillary thyroid carcinomas, which could aid in discriminating between the lesion types. Moreover, among these miRNAs, miR-146-5p as well as miR199a-5p, miR-199b-5p, miR-1285-5p, miR-1915-3p, miR-4516, and miR-148b-3p are associated with the infiltrative growth of follicular variant of papillary thyroid carcinomas. Three downregulated miRNAs, miR-152-3p, miR-185-5p, and miR-574-3p, might facilitate differential diagnosis between NIFTP and follicular adenomas, whereas the differential expression of miR-10a-5p and miR-320e likely helps discriminate between this new entity, NIFTP, and infiltrative follicular variant of papillary thyroid carcinoma.

From a practical point of view, although our findings require further confirmation in future studies, we suggest that a panel of these markers could be used in presurgical fine-needle aspiration because almost all of these lesions are classified as indeterminate nodules.

\section{Acknowledgments}

This work was supported by the Associazione Italiana per la Ricerca sul Cancro (AIRC) (Italy) and Progetti di Ricerca di Ateneo, Università di Pisa (PRA 2016). Grant number and source of support: 549999_AIRC_IRG_10316_2013_Basolo 549999_2015_ BASOLO_PRA2016.

\section{Disclosure/conflict of interest}

The authors declare no conflict of interest.

\section{References}

1 Tan GH, Gharib H. Thyroid incidentalomas: management approaches to nonpalpable nodules discovered incidentally on thyroid imaging. Ann Intern Med 1997;126:226-231.

2 Baloch ZW, LiVolsi VA. Pathology of thyroid gland. In: LiVolsi VA, Asa SL (eds). Endocrine Pathology. Churchill Livingstone: Philadelphia, PA, USA, 2002, p 69.

3 Piana S, Frasoldati A, Di Felice E, et al. Encapsulated well-differentiated follicular-patterned thyroid carcinomas do not play a significant role in the fatality rates from thyroid carcinoma. Am J Surg Pathol 2010;34: 868-872.

4 Vivero M, Kraft S, Barletta JA. Risk stratification of follicular variant of papillary thyroid carcinoma. Thyroid 2013;23:273-279.

5 Kakudo K, Bai Y, Liu Z, et al. Encapsulated papillary thyroid carcinoma, follicular variant: a misnomer. Pathol Int 2012;62:155-160.

6 Liu J, Singh B, Tallini G, et al. Follicular variant of papillary thyroid carcinoma: a clinicopathologic study of a problematic entity. Cancer 2006;107: 1255-1264.

7 Vaccarella S, Dal Maso L, Laversanne M, et al. The impact of diagnostic changes on the rise in thyroid cancer incidence: a population-based study in selected high-resource countries. Thyroid 2015;25: 1127-1136.

8 Davies L, Morris LG, Haymart M, et al. American Association of Clinical Endocrinologists and American College of Endocrinology disease state clinical review: the increasing incidence of thyroid cancer. Endocr Pract 2015;21:686-696.

9 Pitoia F, Jerkovich F, Urciuoli C, et al. Implementing the modified 2009 American Thyroid Association Risk Stratification System in thyroid cancer patients with low and intermediate risk of recurrence. Thyroid 2015;5:1235-1242.

10 Baloch Z, LiVolsi VA, Henricks WH, et al. Encapsulated follicular variant of papillary thyroid carcinoma. Am J Clin Pathol 2002;118:603-605.

11 Ganly I, Wang L, Tuttle RM, et al. Invasion rather than nuclear features correlates with outcome in encapsulated follicular tumors: further evidence for the reclassification of the encapsulated papillary thyroid carcinoma follicular variant. Hum Pathol 2015;46: $657-664$.

12 Nikiforov YE, Seethala RR, Tallini G, et al. Nomenclature revision for encapsulated follicular variant of papillary thyroid carcinoma: a paradigm shift to reduce overtreatment of indolent tumors. JAMA Oncol 2016;2: 1023-1029.

13 Maletta F, Massa F, Torregrossa L, et al. Cytological features of 'noninvasive follicular thyroid neoplasm with papillary-like nuclear features' and their correlation with tumor histology. Hum Pathol 2016;54: 134-142.

14 Esquela-Kerscher A, Slack FJ. Oncomirs-microRNAs with a role in cancer. Nat Rev Cancer 2006;6:259-269.

15 Negrini M, Ferracin M, Sabbioni S, et al. MicroRNAs in human cancer: from research to therapy. J Cell Sci 2007;120:1833-1840.

$16 \mathrm{Li} \mathrm{Y,} \mathrm{Chen} \mathrm{D,} \mathrm{Jin} \mathrm{LU,} \mathrm{et} \mathrm{al.} \mathrm{Oncogenic} \mathrm{microRNA-142-}$ $3 p$ is associated with cellular migration, proliferation and apoptosis in renal cell carcinoma. Oncol Lett 2016;11:1235-1241.

17 Zhou N, Qu Y, Xu C, et al. Upregulation of microRNA-375 increases the cisplatin-sensitivity of human gastric cancer cells by regulating ERBB2. Exp Ther Med 2016;11:625-630.

18 Venkatadri R, Muni T, Iyer AK, et al. Role of apoptosisrelated miRNAs in resveratrol-induced breast cancer cell death. Cell Death Dis 2016;7:e2104.

19 Fan D, Wang Y, Qi P, et al. MicroRNA-183 functions as the tumor suppressor via inhibiting cellular invasion and metastasis by targeting MMP-9 in cervical cancer. Gynecol Oncol 2016;141:166-174. 
20 Petrović N, Kolaković A, Stanković A, et al. miR-155 expression levels indicate its predominant role in breast cancer pathogenesis and lymph-node metastasis in three breast cancer groups. Cancer Biomark 2016;16: 385-394.

21 Yanaihara N, Caplen N, Bowman E, et al. Unique microRNA molecular profiles in lung cancer diagnosis and prognosis. Cancer Cell 2006;9:189-198.

22 Calin GA, Ferracin M, Cimmino A, et al. A MicroRNA signature associated with prognosis and progression in chronic lymphocytic leukemia. N Engl J Med 2005;353: 1793-1801.

$23 \mathrm{He} \mathrm{H}$, Jazdzewski K, Li W. The role of microRNA genes in papillary thyroid carcinoma. Proc Natl Acad Sci USA 2005;02:19075-19080.

24 Pallante P, Visone R, Ferracin M, et al. MicroRNA deregulation in human thyroid papillary carcinomas. Endocr Relat Cancer 2006;13:497-508.

25 Tetzlaff MT, Liu A, Xu X, et al. Differential expression of miRNAs in papillary thyroid carcinoma compared to multinodular goiter using formalin fixed paraffin embedded tissues. Endocr Pathol 2007;18:163-173.

26 Czajka AA, Wójcicka A, Kubiak A, et al. Family of microRNA-146 regulates RAR $\beta$ in papillary thyroid carcinoma. PLoS One 2016;11:e0151968.

27 Braun J, Hoang-Vu C, Dralle H, et al. Downregulation of microRNAs directs the EMT and invasive potential of anaplastic thyroid carcinomas. Oncogene 2010;29: 4237-4244.

28 Colamaio M, Borbone E, Russo L, et al. miR-191 downregulation plays a role in thyroid follicular tumors through CDK6 targeting. J Clin Endocrinol Metab 2011;96:E1915-E1924.

29 Yip L, Kelly L, Shuai Y, et al. MicroRNA signature distinguishes the degree of aggressiveness of papillary thyroid carcinoma. Ann Surg Oncol 2011;18: 2035-2041.

30 DeLellis RA, Williams ED. Tumors of thyroid and parathyroid. In: DeLellis RA, Lloyd RV, Heitz PU (eds). Pathology and Genetics of Tumours of Endocrine Organs. IARC Press: Lyon, France, 2004, pp 49-133.

31 Salvatore G, Giannini R, Faviana P, et al. Analysis of BRAF point mutation and RET/PTC rearrangement refines the fine-needle aspiration diagnosis of papillary thyroid carcinoma. J Clin Endocrinol Metab 2004;89: 5175-5180.

32 Nikiforov YE, Steward DL, Robinson-Smith TM, et al. Molecular testing for mutations in improving the fineneedle aspiration diagnosis of thyroid nodules. J Clin Endocrinol Metab 2009;94:2092-2098.

33 Nikiforov YE, Carty SE, Chiosea SI, et al. Highly accurate diagnosis of cancer in thyroid nodules with follicular neoplasm/suspicious for a follicular neoplasm cytology by ThyroSeq v2 next-generation sequencing assay. Cancer 2014;120:3627-3634.

34 Nikiforov YE, Carty SE, Chiosea SI, et al. Impact of the multi-gene ThyroSeq Next-Generation Sequencing Assay on cancer diagnosis in thyroid nodules with atypia of undetermined significance/follicular lesion of undetermined significance cytology. Thyroid 2015;25: 1217-1223.

35 Nishino M. Molecular cytopathology for thyroid nodules: A review of methodology and test performance. Cancer Cytopathol 2016;124:14-27.

36 Weber F, Teresi RE, Broelsch CE, et al. A limited set of human MicroRNA is deregulated in follicular thyroid carcinoma. J Clin Endocrinol Metab 2006;91:3584-3591.
37 Vriens MR, Weng J, Suh I, et al. MicroRNA expression profiling is a potential diagnostic tool for thyroid cancer. Cancer 2012;118:3426-3432.

38 Guo Z, Hardin H, Montemayor-Garcia C, et al. In situ hybridization analysis of miR-146b-5p and miR-21 in thyroid nodules: diagnostic implications. Endocr Pathol 2015;26:157-163.

39 Dettmer M, Perren A, Moch H, et al. Comprehensive MicroRNA expression profiling identifies novel markers in follicular variant of papillary thyroid carcinoma. Thyroid 2013;23:1383-1389.

40 Chou CK, Chen RF, Chou FF, et al. miR-146b is highly expressed in adult papillary thyroid carcinomas with high risk features including extrathyroidal invasion and the BRAF(V600E) mutation. Thyroid 2010;20: 489-494.

41 Lima CR, Geraldo MV, Fuziwara CS, et al. MiRNA-146b-5p upregulates migration and invasion of different papillary thyroid carcinoma cells. Cancer 2016;16:108.

42 Bhaumik D, Scott GK, Schokrpur S, et al. Expression of microRNA-146 suppresses NF-kappaB activity with reduction of metastatic potential in breast cancer cells. Oncogene 2008;27:5643-5647.

43 Visconti R, Cerutti J, Battista S, et al. Expression of the neoplastic phenotype by human thyroid carcinoma cell lines requires NFkappaB p65 protein expression. Oncogene 1997;15:1987-1994.

44 Pallante P, Battista S, Pierantoni GM, et al. Deregulation of microRNA expression in thyroid neoplasias. Nat Rev Endocrinol 2014;10:88-101.

45 Hébrant A, Floor S, Saiselet M, et al. miRNA expression in anaplastic thyroid carcinomas. PLoS One 2014;9:e103871.

46 Lin Z, Li JW, Wang Y, et al. Abnormal miRNA-30e expression is associated with breast cancer progression. Clin Lab 2016;62:121-128.

47 Feng G, Shi H, Li J, et al. MiR-30e suppresses proliferation of hepatoma cells via targeting prolyl 4hydroxylase subunit alpha-1 (P4HA1) mRNA. Biochem Biophys Res Commun 2016;472:516-522.

48 Cong D, He M, Chen S, et al. Expression profiles of pivotal microRNAs and targets in thyroid papillary carcinoma: an analysis of The Cancer Genome Atlas. Onco Targets Ther 2015;8:2271-2277.

49 Aragon Han P, Weng CH, Khawaja HT, et al. MicroRNA expression and association with clinicopathologic features in papillary thyroid cancer: a systematic review. Thyroid 2015;25:1322-1329.

50 Sadeghian Y, Kamyabi-Moghaddam Z, Nodushan SM, et al. Profiles of tissue microRNAs; miR-148b and miR-25 serve as potential prognostic biomarkers for hepatocellular carcinoma. Tumour Biol 2015. e-pub ahead of print 25 July 2016; doi: 10.1007/s13277-015-3799-y.

$51 \mathrm{Ge} \mathrm{H}$, Li B, Hu WX, et al. MicroRNA-148b is downregulated in non-small cell lung cancer and associated with poor survival. Int J Clin Exp Pathol 2015;8: 800-805.

52 Rehbein G, Schmidt B, Fleischhacker M. Extracellular microRNAs in bronchoalveolar lavage samples from patients with lung diseases as predictors for lung cancer. Clin Chim Acta 2015;450:78-82.

53 Hidaka H, Seki N, Yoshino H, et al. Tumor suppressive microRNA-1285 regulates novel molecular targets: aberrant expression and functional significance in renal cell carcinoma. Oncotarget 2012;3:44-57.

54 Bell EH, Kirste S, Fleming JL, et al. A novel miRNA-based predictive model for biochemical failure 
following post-prostatectomy salvage radiation therapy. PLoS One 2015;10:e0118745.

$55 \mathrm{Li} \mathrm{S}$, Ma Y, Hou X, et al. miR-185 acts as a tumor suppressor by targeting AKT1 in non-small cell lung cancer cells. Int J Clin Exp Pathol 2015;8: 11854-11862.

56 Wang R, Tian S, Wang HB, et al. MiR-185 is involved in human breast carcinogenesis by targeting Vegfa. FEBS Lett 2014;588:4438-4447.
57 Chiyomaru T, Yamamura S, Fukuhara S, et al. Genistein up-regulates tumor suppressor microRNA-574-3p in prostate cancer. PLoS One 2013;8:e58929.

$58 \mathrm{Yu} \mathrm{T}$, Liu L, Li J, et al. MiRNA-10a is upregulated in NSCLC and may promote cancer by targeting PTEN. Oncotarget 2015;6:30239-30250.

59 Perez-Carbonell L, Sinicrope FA, Alberts SR, et al. MiR-320e is a novel prognostic biomarker in colorectal cancer. Br J Cancer 2015;113:83-90. 